\title{
The Potential Distribution due to a Dipole Source in the Fetal Heart: A Numerical Model of the Maternal Abdomen
}

\author{
SHIMON ABBOUD ${ }^{\mathrm{a}, *}$, MICHAEL ARBEL ${ }^{\mathrm{a}}$, MOSHE ROSENFELD $^{\mathrm{b}}$, IZUKA UDOM-RICE ${ }^{\mathrm{c}}$, DAN SKUPSKI $^{\mathrm{c}}$, \\ DEBORAH FRIEDMAN ${ }^{\mathrm{d}}$ and FRANK A. CHERVENAK ${ }^{\mathrm{c}}$ \\ aepartment of Biomedical Engineering, Faculty of Engineering, Tel Aviv University; ${ }^{b}$ Department of Fluid Mechanics, Faculty of \\ Engineering, Tel Aviv University; ${ }^{\mathrm{C}}$ Department of Obstetrics and Gynecology, The New York Hospital, Cornell Medical Center; \\ ${ }^{\mathrm{d}}$ Department of Pediatrics, The New York Hospital, Cornell Medical Center
}

(Received 22 May 1998; Revised II November 1998; In final form 9 February 1999)

\begin{abstract}
The potential distribution in a three dimensional model of the abdomen during pregnancy was studied numerically. Magnetic Resonance Imaging scans were used to construct a three dimensional model with eight different compartments of the abdomen: fetal body, vernix caseosa layer, placenta, uterus fluid, soft tissue, spinal cord, fat and muscles. Each compartment was assigned with its own conductivity and the integral form of the conservation volume conductor equation was solved by a finite volume method. The surface potential distribution of the maternal abdomen was studied for different compartment conductivity and for varying dipole source locations and orientations in the fetal heart. The present study supports the hypothesis that the reduction in the amplitude of the abdominal fetal electrocardiogram around the 30th week of gestation is related to the appearance of a low conductive isolating layer around the fetus. The study shows that an opening in the vernix caseosa layer increases the amplitude of the abdominal fetal ECG with the creation of a preferred signal pathway. It is also shown that the surface potential distribution depends on the fetal posture. Thus, choosing the correct bipolar recording and the appropriate sites of the recording electrodes may result in an increase in the peak to peak amplitude of the fetal ECG.
\end{abstract}

Keywords: Abdominal fetal ECG, numerical model

\section{INTRODUCTION}

Fetal ECG recordings have shown a decrease in amplitude around the 30 th week of gestation. Several studies investigated possible causes for this unexpected observation. Based on fetal ECG recordings at multiple sites of mainly patients near term, Kahn [1] and Roche and Hon [2] suggested that the fetal ECG is conducted to the surface of the abdomen by distinct pathways. Both papers suggested that the oro-nasal cavity of the fetus might serve as a distinct pathway. Kahn [1] further proposed preferential conduction

${ }^{*}$ Corresponding Author: Tel Aviv, 69978, Israel; Tel: 972-3-640-7397; Fax: 972-3-642-7939; E-mail: abboud@eng.tau.ac.il 
through the umbilical cord to the placenta. On the other hand, Taccardi [3] concluded from his observations that the medium between the fetal heart and the maternal abdomen could be described as electrically homogeneous. These studies and others [1-7] suggested two main hypotheses concerning the volume conductor electrical behavior of the abdomen during pregnancy: the uniform volume conductor and the preferred conduction pathway. The fetal ECG recorded from an abdominal lead system showed that prior to 28 weeks of gestation the hypothesis of a uniform volume conductor might be valid, while after 34 weeks the conduction pathway hypothesis should be preferred $[3,4]$. The decrease was related to the appearance of a low conductive isolating layer around the fetus, the vernix caseosa [8]. After 34 weeks of gestation, it was suggested that a preferred signal pathway, possibly through the umbilical cord [1] and the oro-nasal cavities of the fetus [1,2], is created and the abdominal fetal electrocardiogram increases in amplitude. The arrangement of different compartments of abdomen and the posture of the fetus during pregnancy also affect the pathways along which the electrical currents generated by the fetal heart are conducted to the abdominal surface [2].

Oostendorp et al. [5,7,9] studied the electrical conduction of the ECG from the fetal heart to the abdominal surface using a theoretical model of the maternal abdomen during pregnancy. The geometry of the maternal abdomen was determined by echo scanner. The maternal abdomen and the fetal body with an electrically isolated layer were defined. To simulate the situation of current escaping from openings (holes) in the vernix caseosa layer, a model with a source consisting of two monopoles was used. Their simulations, verified by multi-lead recordings of the fetal ECG, showed that early in pregnancy the conduction can be described by an homogeneous model, while in late pregnancy the conduction is dominated by the isolating effect of the vernix caseosa layer.

The motivation for the present study was to investigate the pathways along which the electrical currents generated by the fetal heart are conducted to the surface of the maternal abdomen. A three dimensional numerical model of the abdomen during pregnancy with a dipole source in the fetus heart was employed. The model is based on MRI images to obtain a realistic shape of the geometry and the integral form of the volume conductor conservation equation was solved by a finite volume method. The effect of modifying the electrical and geometrical properties of the tissue between the source and the recording sites on the surface fetal ECG are investigated. The surface potential distribution on the maternal abdomen was also studied for varying dipole source locations and orientations in the fetal heart representing different postures of the fetus.

\section{Methods}

\section{Scanning and image processing of MRI slices}

MRI axial images at a distance of $2.5 \mathrm{~cm}$ from each other were scanned with an HP ScanJet Plus scanner with 16 Gray-levels and 150 dots per inch resolution. Noise reduction using median filtering [10] and contour following algorithm [11] were applied to the image. Seven main compartments were extracted from the image and an isotropic homogeneous conductivity was assigned to each one of them [12-16]. The conductivity of the fetus body, the amniotic fluid, the placenta, the spinal cord bone, the spinal cord muscles, the soft tissue and the fat were $0.40,1.66$, $1.55,0.00041,0.3,0.222,0.333$ Siemens, respectively. The extremities of the fetus are not included in the model. These compartments can be seen in Figure 1 (panel A), where a typical image processed MRI scan is presented. The MRI scans were taken at the 28th week of gestation. An additional layer representing the vernix caseosa around the fetus was added manually. The distribution of this layer around the fetus is not homogenous. The thickness and the conductivity of the vernix caseosa layer can be changed in the numerical model. An opening in this layer next to the fetus head was used to simulate the oro-nasal cavity of the fetus. This opening serves as a preferred pathway along which the electrical currents generated by the fetal heart are conducted to the surface of the maternal abdomen. 


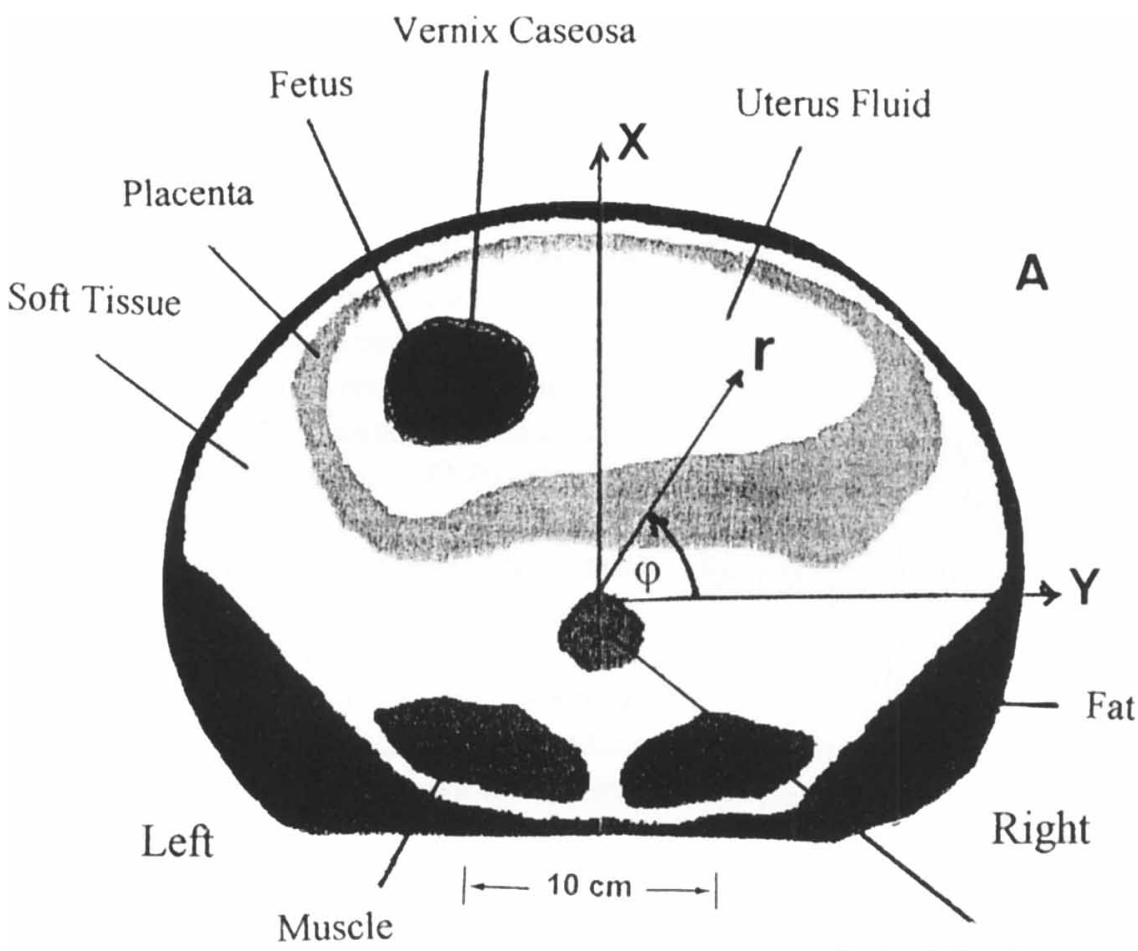

Spinal Cord

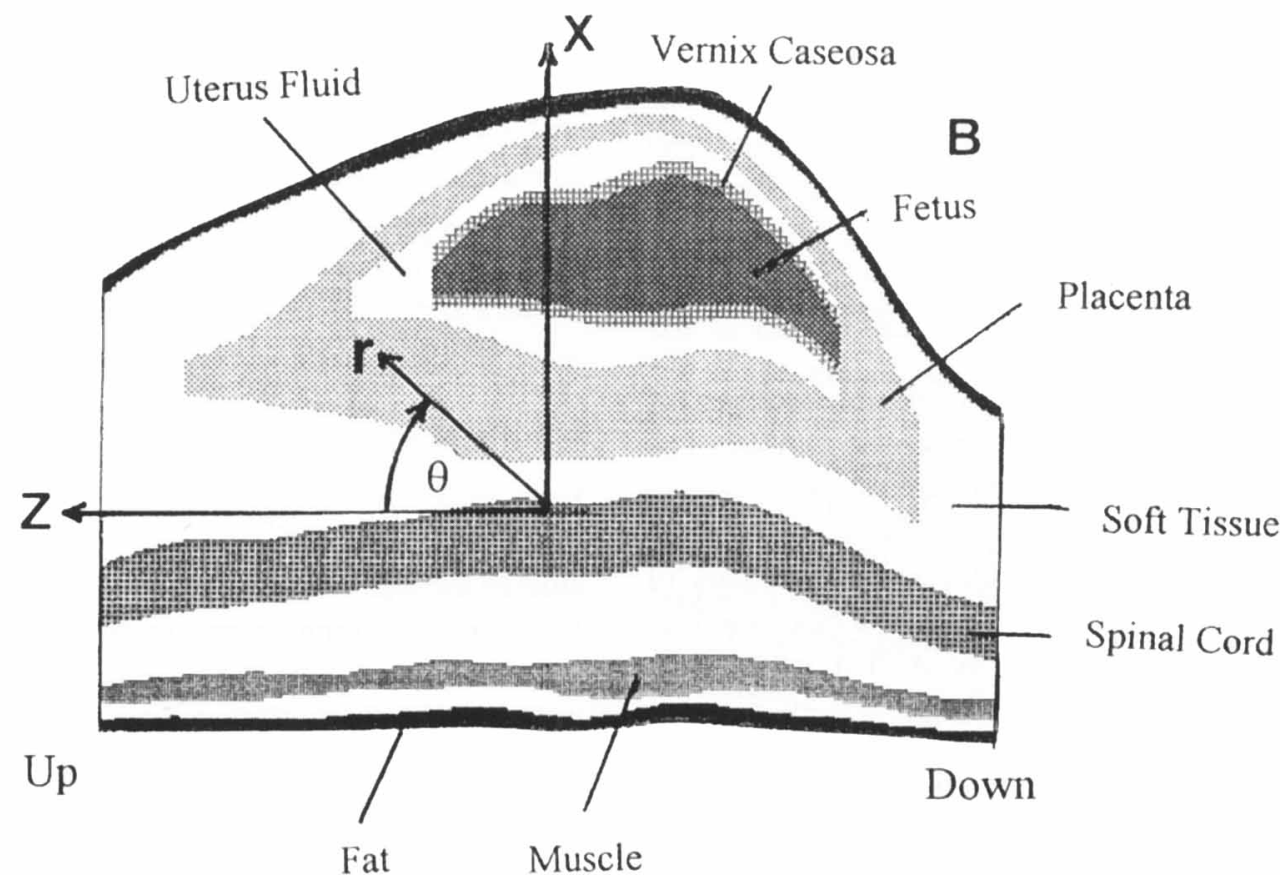

FIGURE 1 The horizontal (panel A) and the sagital (panel B) planes taken from the three dimensional model of the abdomen with the spherical coordinate system $(r, \theta, \phi)$ used. The fetus head, the vernix caseosa layer, the amniotic fluid, the placenta, the spinal cord, the muscles and the fat are shown by the shaded regions. 


\section{Construction of the $3 D$ model from the $2 D$ MRI scans}

In order to construct the 3D abdominal model from the 2D MRI scans, a cubic spline interpolation was used to define mid-section data, including the boundaries between the compartments. The appropriate conductivity is assigned to each compartment. Figure 1 shows the horizontal (panel A) and the sagital (panel B) plane cuts from the 3D model of the abdomen as well as the spherical coordinate system $(r, \theta, \phi ; 0 \leq \theta \leq \pi, 0 \leq \phi \leq 2 \pi)$ used in the computations. The vernix caseosa layer employed in the breech and cephalic positions, respectively, is shown in the sagital plane with openings in the upper and lower parts of the fetus body.

\section{Numerical solution of the potential due to a dipole source in the fetal heart}

The volume conductor problem is assumed to be quasi-static, linear, and the capacitive components of the electric impedance of the body tissues are neglected [17]. The bioelectric sources, which are related with the movement of ions across the cell membrane, are represented as a current source. The potential distribution in the presence of current sources in a control volume $V$ with the face $S$ is given by the Gauss law

$$
\oint_{S} \sigma \nabla \psi \cdot \mathrm{d} S=\int_{V} I \mathrm{~d} V,
$$

where $\sigma$ is the conductivity, $\psi$ is the potential and $I$ is the current volume source density. The boundary conditions specify no current flow into the air, i.e. $\sigma \partial \psi / \partial n=0,(n$ is the normal to the outer boundary). In physiological applications of the volume conductor equation (1), a discontinuous variation of the conductivity $\sigma$ that may be as large as two orders of magnitude is very common because of the presence of several compartments with distinct material properties.

\section{Approximation of the volume conductor equation}

A finite volume method (FVM) for solving a four concentric sphere model of the head to investigate the influence of skull thickness on the scalp potential distribution was developed in our laboratory $[18,19]$. This efficient solver was also used for solving a two dimensional model of the head to investigate the left-right asymmetry of visual evoked potentials in brain damaged patients [20,21]. In a subsequent study, the finite volume methodology was extended to solve the 3-D integral volume conductor equation in general geometry and arbitrary distribution of electrical conductivity [22].

In the FVM, the physical domain is divided into a large number of arbitrary hexahedron cells, known also as the primary cells. The division is performed by generating an appropriate mesh. The faces of each cell are denoted by $\xi, \eta$ and $\zeta$, according to the direction of the curvilinear coordinates. The indices $i, j, k$ are assigned to the center of each cell, while the eight vertices correspond to $i \pm 1 / 2$, $j \pm 1 / 2, k \pm 1 / 2$. The faces of the cells are given by the normal area vectors $\boldsymbol{S}^{l}$, where $l=(\xi, \eta$ or $\zeta)$. The discrete potential function unknowns $\psi_{i, j, k}$ are defined at the center of the cells (not at the vertices as in most discretization methods) to simplify the calculation of the fluxes.

The Gauss law (1) is discretized for each primary cell. It is approximated by the second-order-accurate scheme

$$
\begin{aligned}
& \left(\sigma \nabla \psi \cdot S^{\xi}\right)_{i+1 / 2, j, k}-\left(\sigma \nabla \psi \cdot S^{\xi}\right)_{i-1 / 2, j, k} \\
& \quad+\left(\sigma \nabla \psi \cdot S^{\eta}\right)_{i, j+1 / 2, k}-\left(\sigma \nabla \psi \cdot S^{\eta}\right)_{i, j-1 / 2, k} \\
& \quad+\left(\sigma \nabla \psi \cdot S^{\zeta}\right)_{i, j, k+1 / 2} \\
& \quad-\left(\sigma \nabla \psi \cdot S^{\zeta}\right)_{i, j, k-1 / 2}=(I V)_{i, j, k}
\end{aligned}
$$

Each term in the left-hand-side represents the current flow through the relevant face, while the right-handside is the current volume source in the cell. The boundary condition (zero current flow) is applied by setting to zero the appropriate current flow term in Equation (2).

The gradient for each face of the primary cell is calculated from the integral definition

$$
\nabla \psi=\frac{1}{V} \oint_{S} \psi \mathrm{d} S
$$

A spherical coordinate system $r, \theta, \phi$ (Figure 1) with equally spaced intervals was employed in the 
present calculations. The mesh included $91 \times 70 \times$ 72 points in the $r, \theta, \phi$ directions; respectively, resulting in a total of 460,000 mesh points. The resolution in the radial direction was $1 \mathrm{~mm}$, while along $\theta$ and $\phi$ an increment of $2.6^{\circ}$ and $5^{\circ}$ was employed, respectively. The discrete equations are solved by the iterative successive over relaxation method. The optimal over-relaxation parameter is selected by a trial and error procedure. The convergence criterion, based on the residual, is $10^{-6}$.

The implementation of Equation (2) for bioelectric cases is confronted with several problems. The abrupt change of the conductivity poses difficulties in the discretization and in the numerical solution of the volume conductor equation because of the discontinuity of the potential gradient. Also, the Neumann conditions specified on the outer boundary result in a singular algebraic system of equations that requires the satisfaction of a discrete compatibility condition to ensure the existence of a solution. Finally, the current flow near geometric singularities (such as singular lines or points) should be calculated differently. The treatment of these issues in the present finite volume method is elaborated in [18,22].

Several cases were solved, differing in the thickness, conductivity and shape of the vernix caseosa layer and in the location and orientation of the dipole source in the fetal heart. The cases include:

1. Models without a vernix caseosa layer, with a closed vernix caseosa layer and a vernix caseosa layer with an opening in the vicinity of the fetus head.

2. Models with varying dipole location and orientation in the fetal heart.

3. Models with varying conductivity of the vernix caseosa layer (but with a constant $3 \mathrm{~mm}$ in thickness).

4. Models with a vernix caseosa layer of varying thickness (but with a constant conductivity of $1.4 \times 10^{-6}$ Siemens).

\section{RESULTS}

Figure 2 shows the potential distribution on the abdominal surface for three cases simulating the cephalic posture with a radial dipole in the fetal heart. In panel $\mathbf{A}$, the potential is shown for a model without a vernix caseosa layer, while in panels $B$ and $\mathrm{C}$, a model with a closed vernix caseosa layer and a model with an opening in the vernix caseosa layer in the vicinity of the fetus head is shown, respectively. In the model with the closed vernix caseosa layer, the peak surface potential amplitude reduces to 0.40 arbitrary units (AU) compared to $5.78 \mathrm{AU}$ in the model without the layer. Introducing an opening in the vernix caseosa layer increases the amplitude to $0.50 \mathrm{AU}$ (a $25 \%$ increase compared to the model with the closed layer).

Arbitrary units (AU), rather than physical units, are employed in the present work. Several considerations led to this choice. The magnitude of the current source density is unknown. On the other hand, the magnitude of the potential depends linearly on the current source density. Therefore, the potential is defined up to an arbitrary factor. The value of this factor is irrelevant since in the present study we are comparing values (e.g. preferred recording sites) rather than being interested in absolute values. Consequently, arbitrary units are satisfactory.

Figure 3 shows for the three models of Figure 2 the potential on a horizontal cross-section through the radial dipole. In all three panels the increment between the potential contour lines is $0.15 \mathrm{AU}$. On the boundaries between the compartments, the potential is continuous but there is a discontinuity in the slope due to the different conductivity. Yet, the flux $\sigma \partial \psi / \partial r$ is continuous because the current source is continuous across the boundaries of the compartments. The potential amplitude as well as its gradient (that is proportional to the current) are larger for the model without the vernix caseosa layer (panel A). The amplitude decreases in the model of the vernix caseosa layer with an opening (panel C), and it becomes very small in the model of the closed vernix caseosa layer (panel B). This is due to the fact that the high resistance vernix caseosa layer attenuates the potential significantly. The current density is distributed according to the tissue resistivity. Consequently, it becomes larger in the opening region, where the resistance is low (high conductivity). 

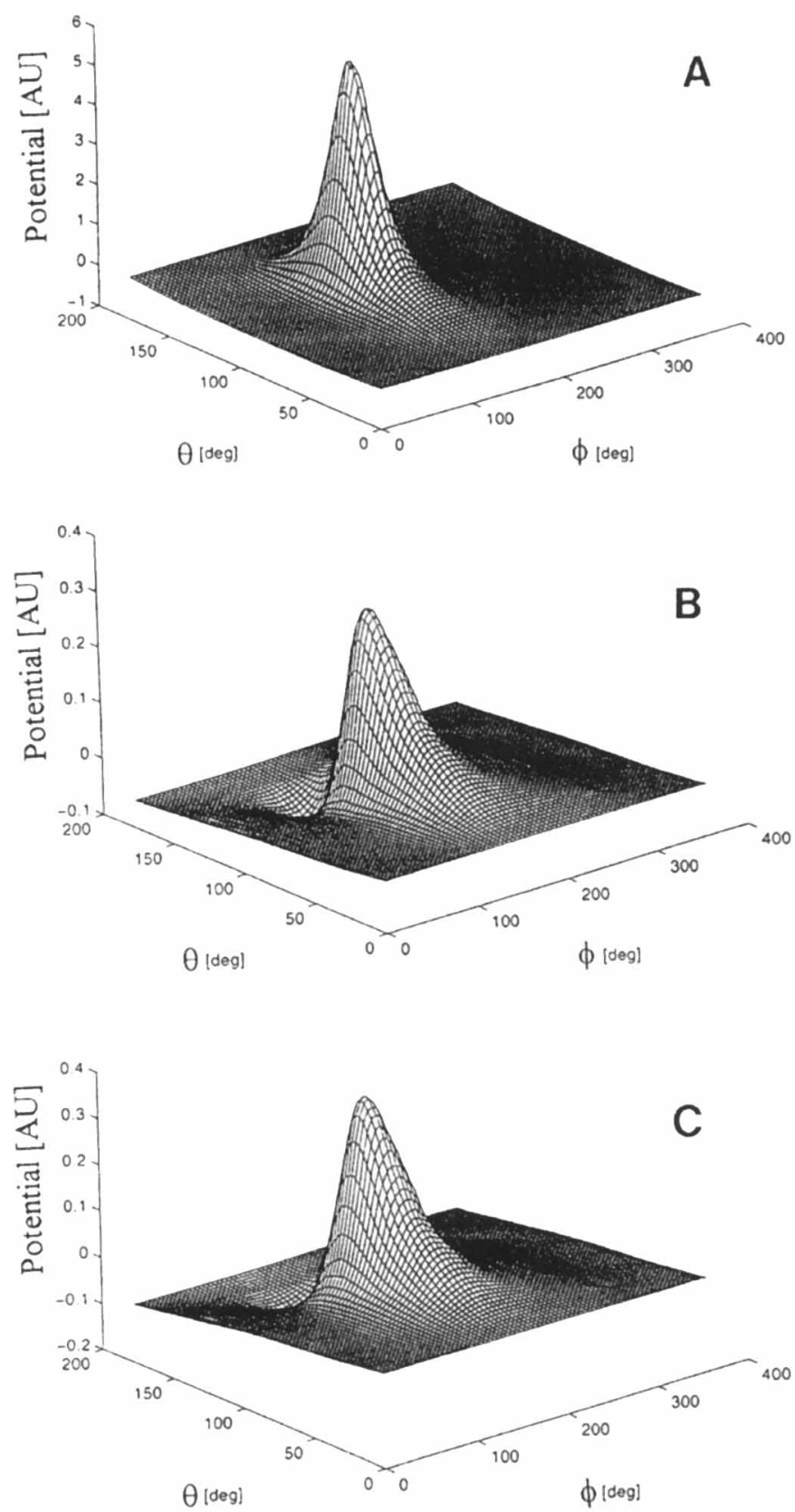

FIGURE 2 The surface potential distribution in three models of the abdomen: a model without a vernix caseosa layer (panel A), a model with a closed layer (panel B) and a model with an opening in the vernix next to the fetus head (panel C). The radial dipole is located in the lower part of the fetus representing the cephalic position.

Table I presents the surface potential peak amplitude for the case with an opening in the vernix caseosa layer. The model was solved for a conductivity of the vernix caseosa layer in the range of $0.7 \cdot 10^{-6}$ to $14 \cdot 10^{-6}$. The surface potential is insensitive to the conductivity of the vernix caseosa layer. Variation of the vernix caseosa layer conductivity by a factor of 20 changed the surface 

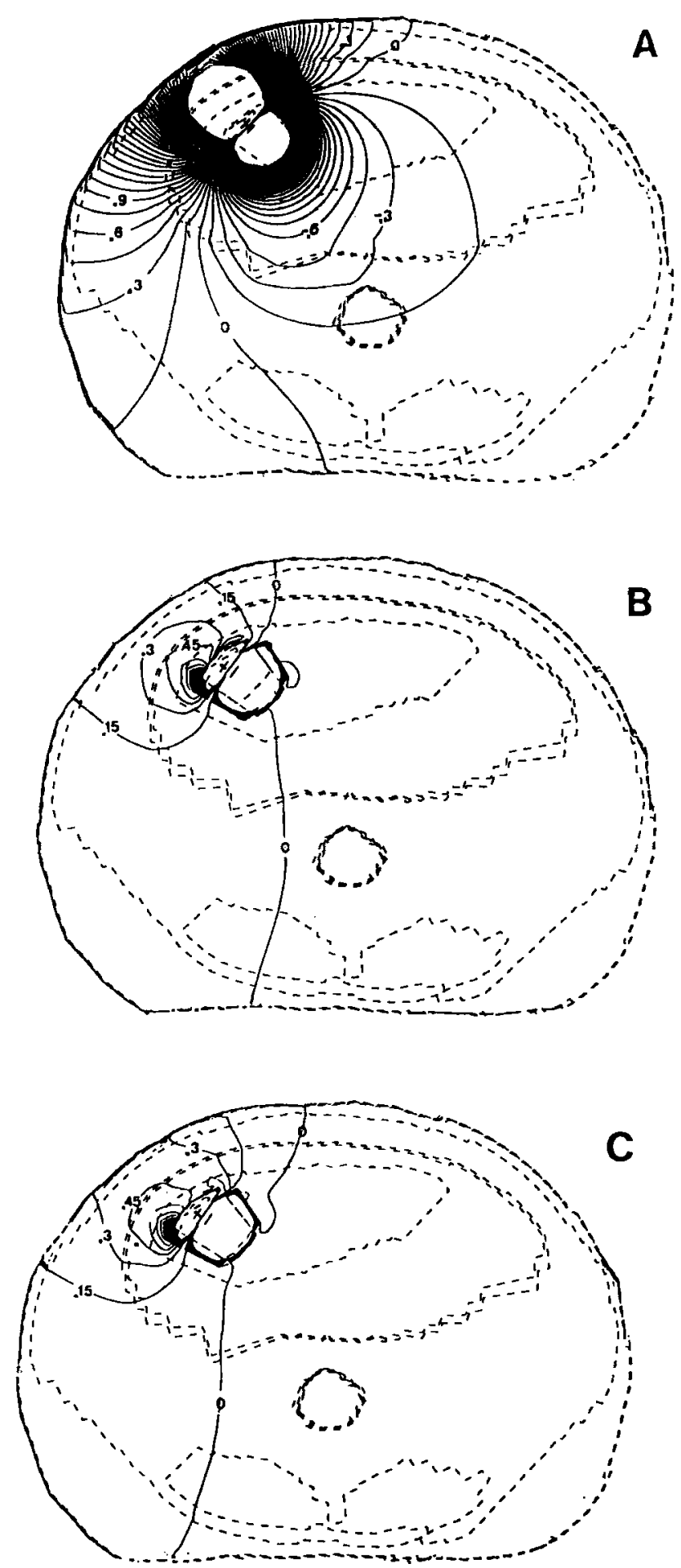

FIGURE 3 The potential distribution in a horizontal cross-section through the radial dipole in the fetal heart for the three models presented in Figure 2. 
TABLE I The effect of Vernix Caseosa conductivity on the surface potential in a model with a radial dipole $\left(\sigma=1.4 \cdot 10^{-6}\right.$ Siemens $)$

\begin{tabular}{clc}
\hline $\begin{array}{c}\text { Vernix } \\
\text { Conductivity } \\
\text { [Siemens] }\end{array}$ & $\begin{array}{c}\text { Potentia] } \\
\text { [AU] }\end{array}$ & $\begin{array}{c}\text { Relative } \\
\text { Potential } \\
{[\%]}\end{array}$ \\
\hline $0.5 \sigma$ & 0.502823 & 99.987 \\
$\sigma$ & 0.50289 & 100.000 \\
$2 \sigma$ & 0.503049 & 100.031 \\
$4 \sigma$ & 0.503352 & 100.091 \\
$10 \sigma$ & 0.50426 & 100.272 \\
\hline
\end{tabular}

potential amplitude less than $0.1 \%$. Figure 4 plots the peak amplitude of the surface potential (together with a decayed exponential best fit curve) for the cases of varying thickness of the vernix caseosa layer (the conductivity is $1.4 \cdot 10^{-6}$ Siemens in all the cases). Variations in the thickness of the vernix layer significantly affect the potential amplitude. A peak amplitude of 5.78 AU was found on the surface potential for the abdominal configuration without a vernix caseosa layer). Adding a 2 or $3 \mathrm{~mm}$ layer of vernix caseosa with the same conductivity as the amniotic fluid (1.6 Siemens) reduces the amplitude to 1.1 and $0.40 \mathrm{AU}$, respectively.

Abdominal models with different postures of the fetus were also solved. The fetus posture is modeled by changing the location and orientation of the dipole source. A dipole source was located in the upper and lower regions of the fetus, representing cephalic and breech positions of the fetus,

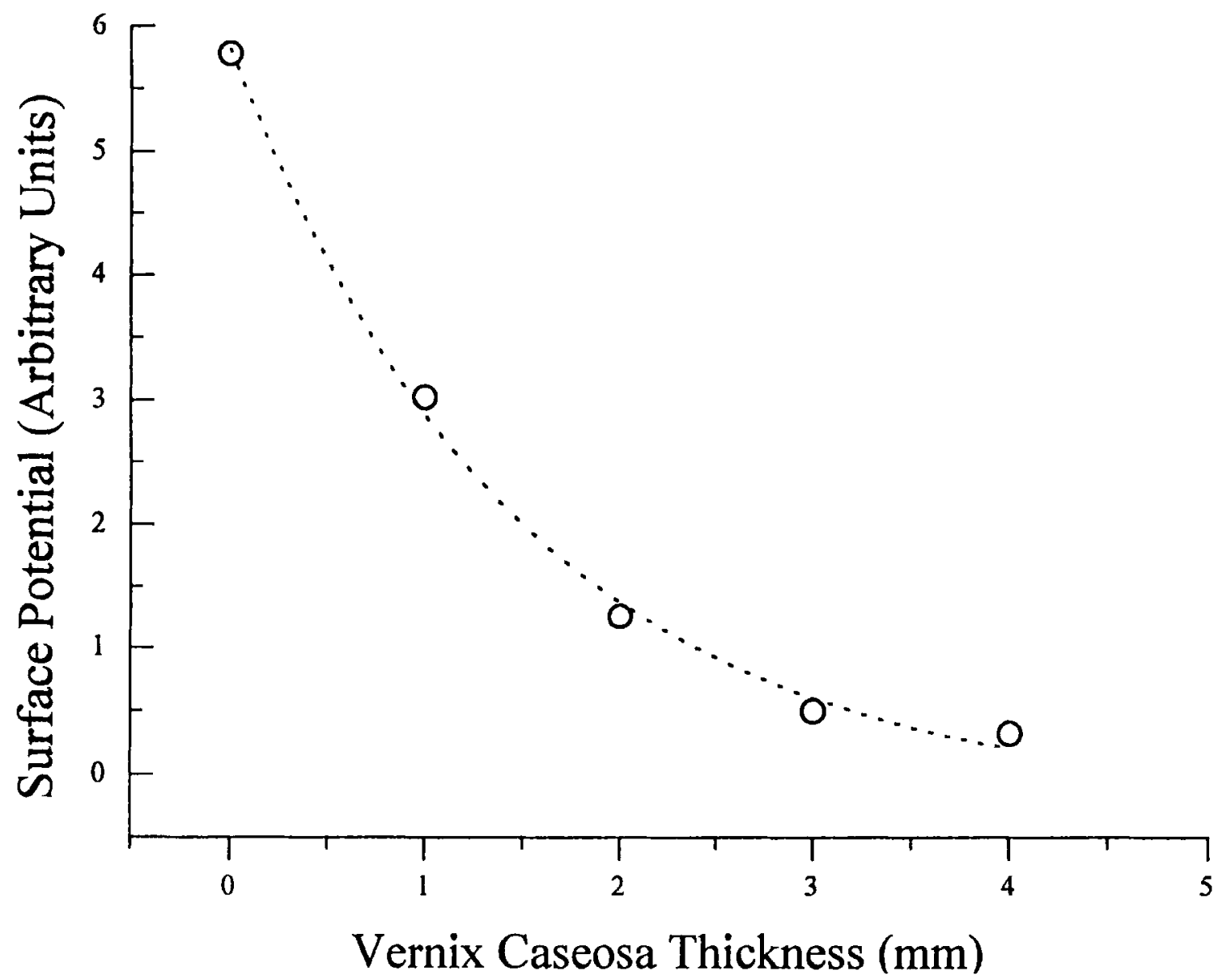

FIGURE 4 The peak surface potential amplitude for varying thickness of the vernix caseosa layer with a radial dipole (the conductivity of the layer is $1.4 \cdot 10^{-6}$ Siemens). The dotted line is the exponential best fit curve. 

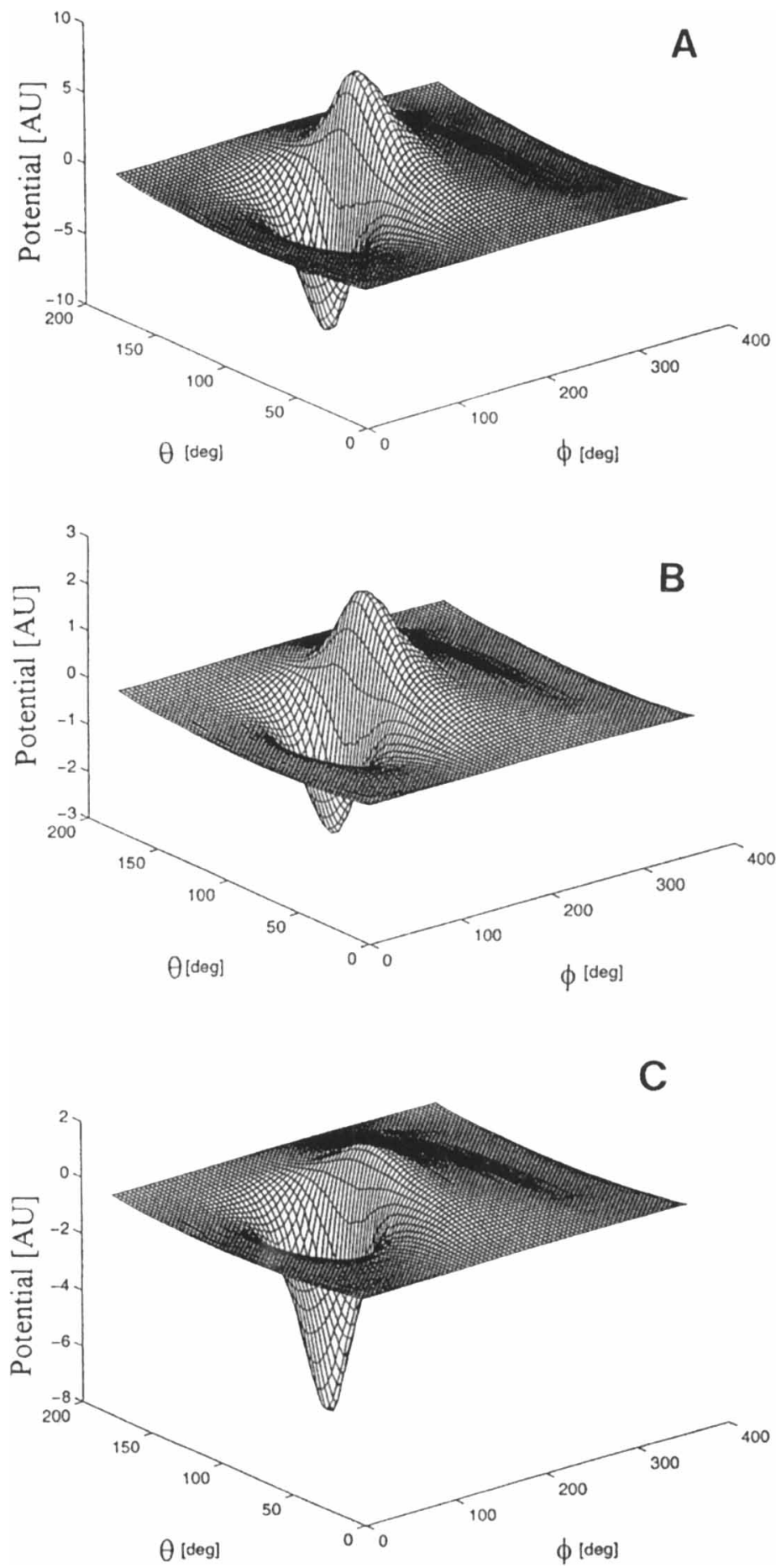

FIGURE 5 The surface potential distribution for three models: a model without the vernix caseosa layer (panel A), a model with a closed layer (panel B) and a model with an opening in the vernix (panel C). A tangential dipole is located in the lower part of the fetus representing the cephalic position. 

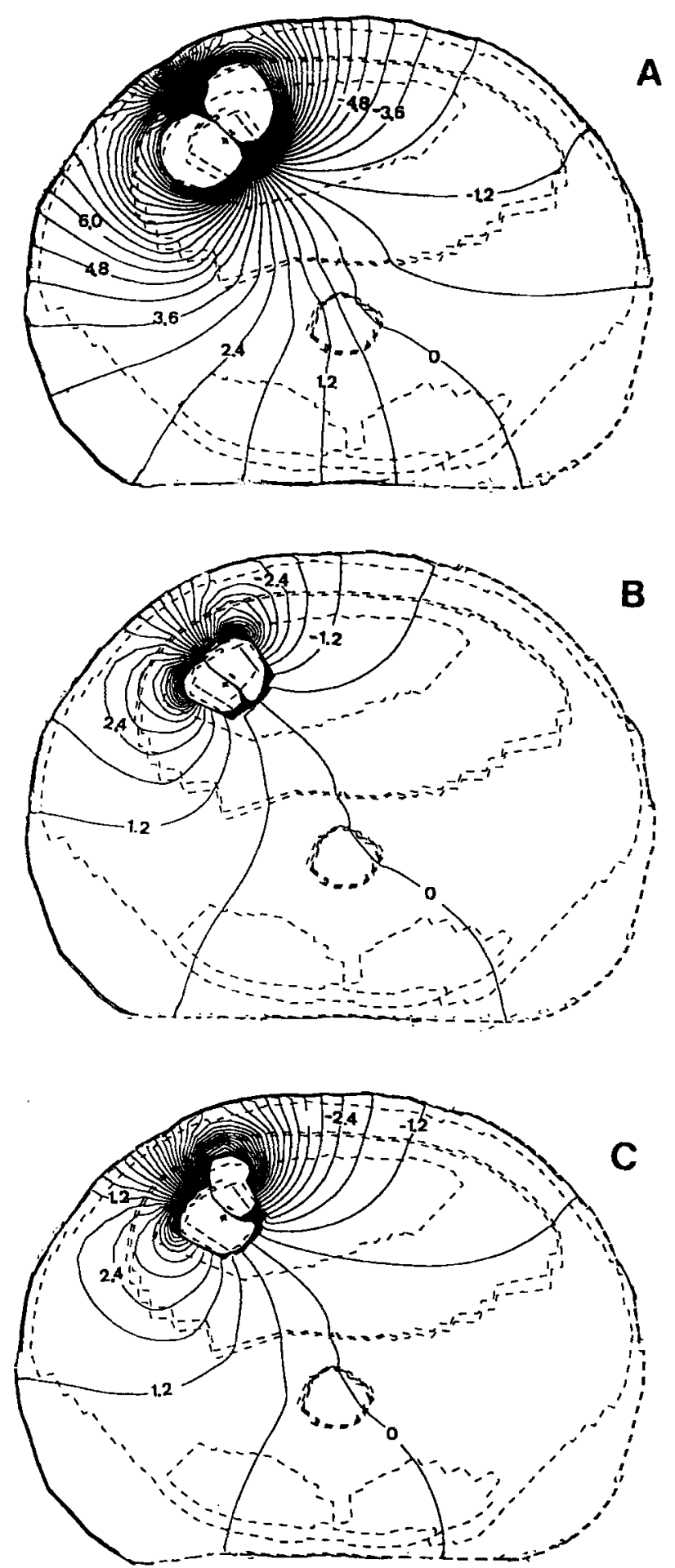

FIGURE 6 The potential distribution in a horizontal cross-section through the tangential dipole of the three models presented in Figure 5. 


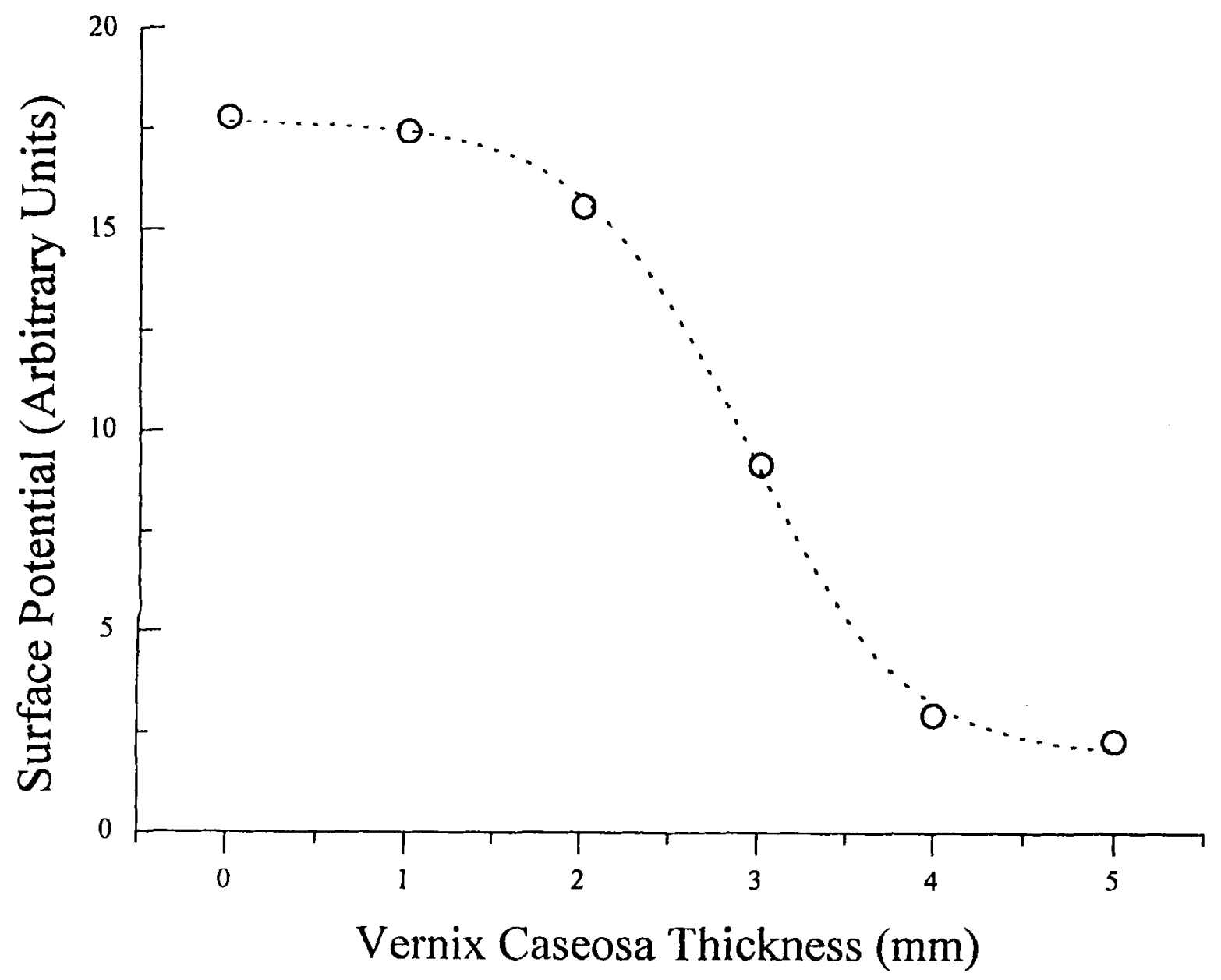

FIGURE 7 The peak surface potential amplitude for varying thickness of the vernix caseosa layer with a tangential dipole (the conductivity of the layer is $1.4 \cdot 10^{-6}$ Siemens). The dotted line is the exponential best fit curve.

TABLE II The effect of Vernix Caseosa conductivity on the surface potential in a model with a tangential dipole ( $\sigma=1.4 \cdot 10^{-6}$ Siemens)

\begin{tabular}{ccr}
\hline $\begin{array}{c}\text { Vernix } \\
\text { Conductivity } \\
\text { [Siemens] }\end{array}$ & $\begin{array}{c}\text { Potential } \\
\text { [AU] }\end{array}$ & $\begin{array}{c}\text { Relative } \\
\text { Potential } \\
{[\%]}\end{array}$ \\
\hline $0.5 \sigma$ & 9.16582 & 99.999 \\
$\sigma$ & 9.16586 & 100.000 \\
$2 \sigma$ & 9.16593 & 100.001 \\
$4 \sigma$ & 9.16607 & 100.002 \\
$10 \sigma$ & 9.16650 & 100.007 \\
\hline
\end{tabular}

respectively. Tangential dipole orientations were used to simulate certain posture positions such as right and left anterior, or right and left posterior.
Figures 5, 6, 7 and Table II present the results obtained for the cases simulating the cephalic posture with a tangential dipole. Figure 5 depicts the surface potential distribution. For the case without the vernix caseosa layer (panel A), a biphasic potential distribution with a peak-to-peak amplitude of $17.80 \mathrm{AU}$ was obtained with a positive peak amplitude of $7.93 \mathrm{AU}$ at the lower-left part of the abdomen, and a negative peak amplitude of $-9.87 \mathrm{AU}$ at the top-right part of the abdomen. In the model with the closed vernix caseosa layer (panel B) a biphasic surface potential distribution is obtained with a smaller peak-amplitude of $4.97 \mathrm{AU}$. Adding an opening in the vernix caseosa layer 
(panel C) changes the potential into a more monophasic distribution with a negative peak amplitude of $-8.5 \mathrm{AU}$ on the lower-left region of the abdomen and a small positive peak on the top-right region (a $85 \%$ increase compared to the model with the completely closed layer).

Figure 6 shows the potential distribution in a horizontal cross-section through a tangential dipole in the fetal heart. The potential distribution is shown for three cases: (i) a model without a vernix caseosa layer (panel A), (ii) a model with a closed vernix caseosa layer (panel B) and (iii) a model with an opening in the vernix caseosa layer (panel C).

Table II presents the dependence of the peak surface potential amplitude on the conductivity of the cases with an opening in the vernix caseosa layer, corresponding to the results presented in Table I for a tangential dipole. In the present case, an even smaller sensitivity of the surface potential to the conductivity of the vernix caseosa layer is found, proving that the conductivity of the layer has no effect, independent of the orientation of dipole. Figure 7 shows the dependence of the peak surface potential amplitude on thickness of the vernix caseosa layer for the tangential dipole cases (the conductivity is $1.4 \cdot 10^{-6}$ Siemens in all the cases). The best fit to a sigmoidal (Boltzman) curve is also shown by the dotted line. The thickness of the layer has a pronounced effect on the surface potential, similar to the findings for a radial dipole (see Figure 6). For example, adding a $3 \mathrm{~mm}$ layer of vernix caseosa reduces the potential amplitude to 9.17 $\mathrm{AU}$ from a value of 17.8 $\mathrm{AU}$ in the absence of the layer. Similar results were obtained for the cases simulating the breech position.

Figure 8 shows an example of a typical fetal ECG waveforms extracted at the 39th week of gestation using an improved data acquisition system developed in our laboratory [23-26]. The recording was performed from three leads: two unipolar leads on the left and right side of the abdomen (Panels $\mathrm{A}$ and $B$, respectively), and a bipolar lead between the two sides of the abdomen. A negative QRS amplitude of $8 \mu$ Volt was recorded on the left side (panel A) and smaller positive amplitude of $2 \mu$ Volt on the right side. For the bipolar lead, positive QRS amplitude of $12 \mu$ volt was recorded. These results are in agreement with the results of the present model with an opening in the vernix caseosa layer (Figure 5 panel C) showing a negative peak amplitude on the lower-left region of the abdomen and a small positive peak on the top-right region of the abdomen. According to the QRS amplitudes obtained at this stage of pregnancy, one AU of the amplitude used in the present model is approximately equal to $1 \mu$ Volt.

\section{DISCUSSION}

Bolte [8] studied the variability of the fetal ECG waveforms throughout the second half of gestation. He found that the amplitude of the fetal ECG decreases rapidly between 26 and 30 weeks of gestation. Subsequently, the fetal ECG magnitude increases gradually until term. Oldenburg and Macklin [4] employed vector-cardiograms recordings from the fetus in utero to study the fetal ECG signal transmission to the maternal abdominal surface. They suggested that a uniform conduction of fetal ECG currents exists between 20 and 28 weeks of gestation and that preferred signal pathway appears to develop after 34 weeks of gestation. Oostendorp et al. [7] constructed a model of the maternal abdomen with an isolating layer around the fetus to estimate the magnitude of the fetal ECG in the case that the fetus is completely covered by an electrically isolating layer of vernix. Employing a dipole source at the fetal heart position, they found that the maximum potential difference at the maternal abdomen was below the detection level of the recording equipment $(5 \mu \mathrm{V})$ throughout the $\mathrm{QRS}$ interval.

The present numerical study demonstrated that the appearance of an isolating vernix caseosa layer with a conductivity of $1.4 \cdot 10^{-6}$ Siemens causes a significant reduction in the surface potential amplitude. For example, a vernix caseosa layer of $1 \mathrm{~mm}$ thickness reduces the surface potential amplitude by as much as $50 \%$. It is in agreement with the experimental results of Bolte et al [8] that show a decrease 

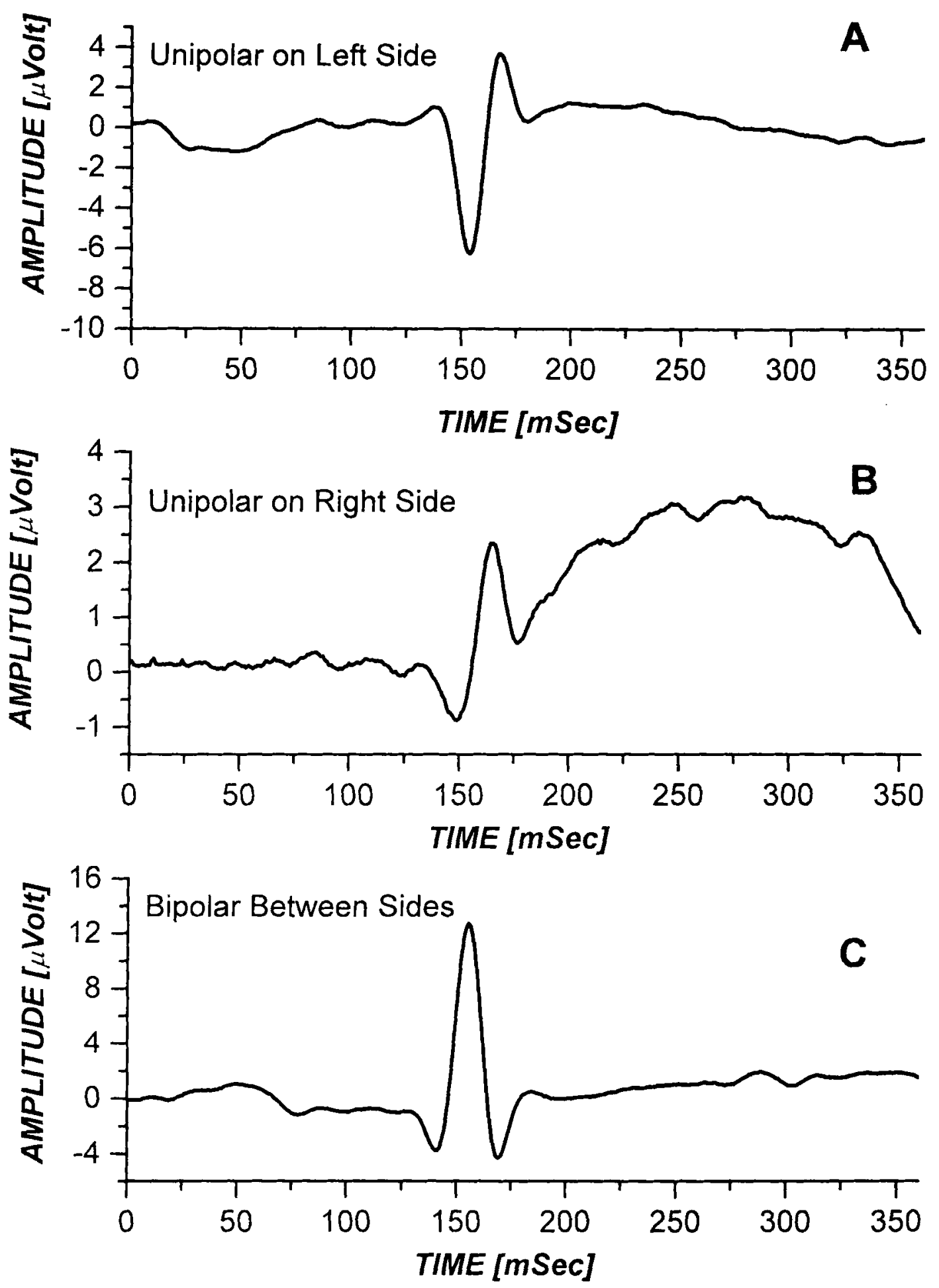

FIGURE 8 An example of a typical fetal ECG waveforms extracted at the $39^{\text {th }}$ week of gestation from three leads: two unipolar leads on the left and right side of the abdomen (Panel A and B, respectively), and a bipolar lead between the two sides of the abdomen (Panel C). 
in the fetal QRS amplitude from $20 \mu \mathrm{V}$ to $10 \mu \mathrm{V}$ between 26 and 30 weeks of gestation. A change in the thickness of this layer from 2 to $3 \mathrm{~mm}$ causes a reduction of $40 \%$ to $60 \%$ in the amplitude of the surface potential, depending on the orientation of the dipole. These observations suggest that the rapid reduction in the fetal ECG magnitude between 26 and 30 weeks of gestation [8] may be attributed to changes in the thickness of the vernix caseosa layer. The conductivity of the vernix caseosa layer, however, does not affect the amplitude of the surface potential (less than $0.1 \%$ for a change of the conductivity by a factor of 20 ).

A biphasic potential distribution is found on the surface of the abdomen in the cases with tangential dipoles (with or without a closed vernix caseosa layer). Adding an opening in the vernix caseosa layer in the vicinity of the fetus head modifies the potential distribution significantly. The potential changes into a more monophasic distribution and the peak amplitude increases. The current density increases in the region of the opening in the vernix caseosa. The larger conductivity in this region significantly modifies the current distribution in the volume conductor, creating a preferred signal pathway through the opening, see Figures 3 and 6 . These results are in agreement with the suggestion of Oostendorp et al. [7] that as pregnancy progresses, gaps appear in the vernix layer and the magnitude of the surface potential increases.

However, the major cause for the gradual increase in amplitude of the surface fetal ECG during pregnancy is probably the growth of the fetal heart and the accompanying strengthening of the ECG source. Moreover, as pregnancy progresses, the fetus increases in size and the distribution of amnionic fluid becomes less uniform. Consequently, it can be speculated that the close proximity of the fetal head to the uterine wall and the high conductivity of the opening in the vernix affect the current distribution near the surface, increasing the peak potential amplitude.

In the present study, it has been also shown that the posture of the fetus, modeled by the location and orientation of the fetal heart dipole, significantly affects the peak amplitude of the surface potential and its location. Tangential dipoles cause negative and positive potential peak amplitudes with peak to peak amplitude almost five time larger than in the case of a radial dipole. This is in agreement with the observations of Roche and Hon [2] who found significant correlation with the fetal presentation.

In conclusion, the present study supports the hypothesis that the reduction in the amplitude of the abdominal fetal electrocardiogram around the 30 th week of gestation is related to the appearance of a low conductive isolating layer around the fetus. It has been shown that the increase in the potential amplitude as pregnancy progresses might be a result of a preferred signal pathway. The present study also shows that the surface potential distribution is significantly affected by the fetal posture. Therefore, selecting the correct bipolar recording with the appropriate sites of the recording electrodes might result in an increase in the peak to peak amplitude of the fetal ECG. The present study clearly shows that such a preferable location of the electrodes does exist for each case studied separately.

\section{References}

[1] Kahn, A. R. (1965). Transmission characteristics in fetal electrocardiography, Proceedings of the Sixteenth Annual Conference on Engineering, Medicine, and Biology, 5, 134.

[2] Roche, J. B. and Hon, E. H. (1965). The fetal electrocardiogram, V. Comparison of lead system, Am. J. Obstet. Gynecol., 92, 1149-1159.

[3] Taccardi, B. (1971). The choice of leads in fetal electrocardiography. Symposium on fetal evolution during pregnancy and labor. Eds. PG Crosignani and G pardi (New York: Academic Press), pp. 211-220.

[4] Oldenburg, J. T. and Macklin, M. (1977). Changes in conduction of the fetal electrocardiogram to the maternal abdominal surface during gestation. Am. J. Obstet. Gynecol., 129, 425-433.

[5] Oostendorp, T. F., van Oosterom, A., Jongsma, H. W. and van Dongen Pieter, W. J. (1986). The potential distribution generated by the fetal heart at the maternal abdomen, $J$. Perinat. Med., 14, 435-444.

[6] Oostendorp, T. F., van Oosterom, A. and Jongsma, H. W. (1989). The fetal ECG throughout the second half of gestation. Clin. Phys. Physiol. Meas, 10(2), 147-160.

[7] Oostendorp, T. F., van Oosterom, A. and Jongsma, H. W. (1989). The effect of changes in the conductive medium on the fetal ECG throughout gestation. Clin. Phys. Physiol. Meas., 10(Suppl B), 11-20.

[8] Bolte, A., Bachman, K. and Kuhn, G. (1966). Die fetalen Herzaktion Potentiale und ihre diagnostiche Bedeutung. Arch. Gynakol, 203, 133-163. 
[9] Oostendorp, T. F. and van Oosterom, A. (1989). Source parameter estimation in inhomogeneous volume conductors of arbitrary shape, IEEE Trans. on Biomed. Eng., 36(3), March.

[10] Atkinson, K. E. (1989). An introduction to numerical analysis, John Wiley and Sons, N.Y.

[11] Dura, R. O. and Hart, P. E. (1973). Pattern Recognition and Scene Analysis, John Wiley and Sons, N.Y.

[12] Oostendorp, T. F., van Oosterom, A. and Jongsma, H. W. (1989). Electrical properties of tissues involved in the conduction of foetal ECG, Med. and Biol. Eng. and Comput., 27, 322-324

[13] Geddes, L. A. and Baker, L. E. (1967). "The specific resistance of biological material - a compendium of data for the biomedical engineer and physiologist", Med. Biol. Eng., 5, $271-293$.

[14] Schwan, H. P. and Kay, C. F. (1957). "The conductivity of living tissues", Ann. N.Y. Acad. Sci., 65, 1007-1013.

[15] Ranck, J. B. and BeMent, S. L. (1965). "The specific impedance of the dorsal columns of cat: an anisotropic medium", Exp. Neurol., 11, 451-463.

[16] Rush, S., Abildskov, J. A. and McFee, R. (1963). "Resistivity of body tissues at low frequencies", Cir. Res., 12, 40.

[17] Plonsey, R. (1969). Bioelectric Phenomena, McGraw-Hill, New York.

[18] Abboud, S., Eshel, Y., Levi, S. and Rosenfeld, M. (1994). Numerical calculation of the potential distribution due to dipole source in a spherical model of the head, Comp. Biomed. Res., 27, 441-455.

[19] Eshel, Y., Levy, S., Rosenfeld, M. and Abboud, S. (1995). Correlation between skull thickness asymmetry and scalp potential estimated by a numerical model of the head. IEEE Trans. Biomed. Eng., 42(3), March, 242-249.

[20] Abboud, S., Bar, L., Rosenfeld, M., Ring, H. and Glass, I. (1996). Left-right asymmetry of visual evoked potentials in brain damaged patients - a mathematical model and experimental results. Ann. Biomed. Eng., 24(1), 75-86.

[21] Abboud, S., Luzon, Y. and Rosenfeld, M. (1996). Effect of Source Location on the Scalp Potential Asymmetry in a Numerical Model of the Head. IEEE Trans Biomed Eng., 43(7), July, 690-696.

[22] Rosenfeld, M., Tanami, R. and Abboud, S. (1996). Numerical solution of the potential distribution due to dipole source in volume conductor with arbitrary geometry and distribution of conductivity. IEEE Trans. Biomed. Eng., 43(7), July, $679-689$.

[23] Abboud, S. and Beker, A. (1989). An improved detection algorithm in fetal electrocardiography. Journal of Electrocardiography, 22, 283-242.

[24] Abboud, S., Barkai, G., Mashiach, S. and Sadeh, D. (1990). Quantification of the fetal electrocardiogram using averaging technique. Computers in Biology and Medicine, 20(3), 147-155.

[25] Abboud, S., Alaluf, A., Einav, S. and Sadeh, D. (1992). Real time abdominal fetal ECG recording using a hardware correlator. Computers in Biology and Medicine, 22(5), $325-335$.

[26] Budin, N. and Abboud, S. (1994). Real Time Multichannel Abdominal Fetal ECG Monitor Using Digital Signal CoProcessor. Computers in Biology and Medicine, 24(6), 451-462. 


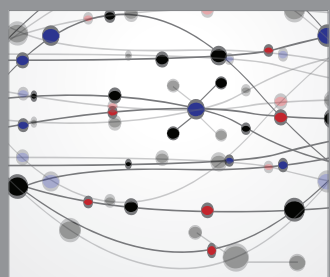

The Scientific World Journal
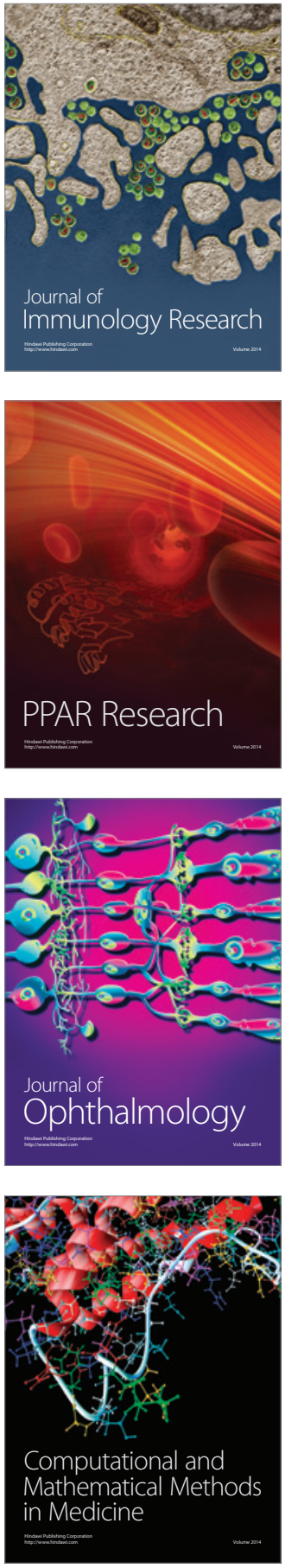

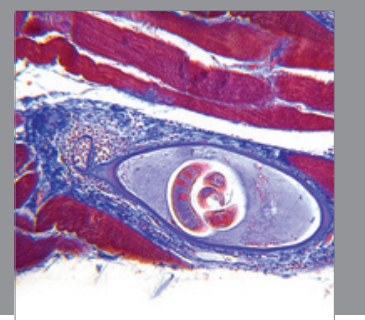

Gastroenterology

Research and Practice
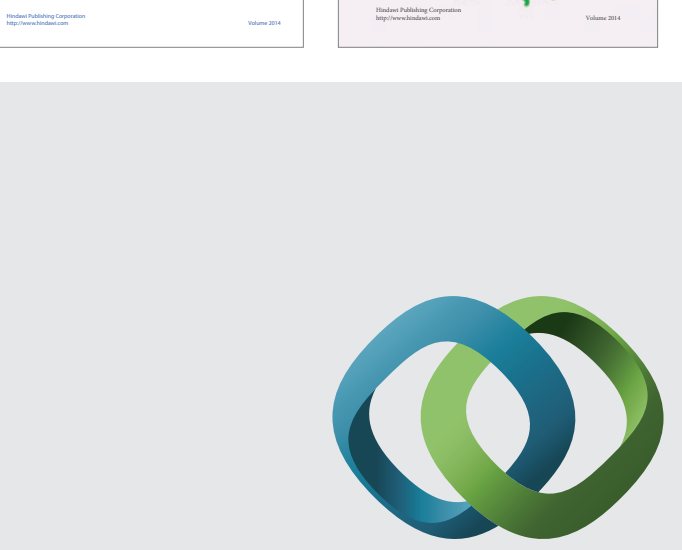

\section{Hindawi}

Submit your manuscripts at

http://www.hindawi.com
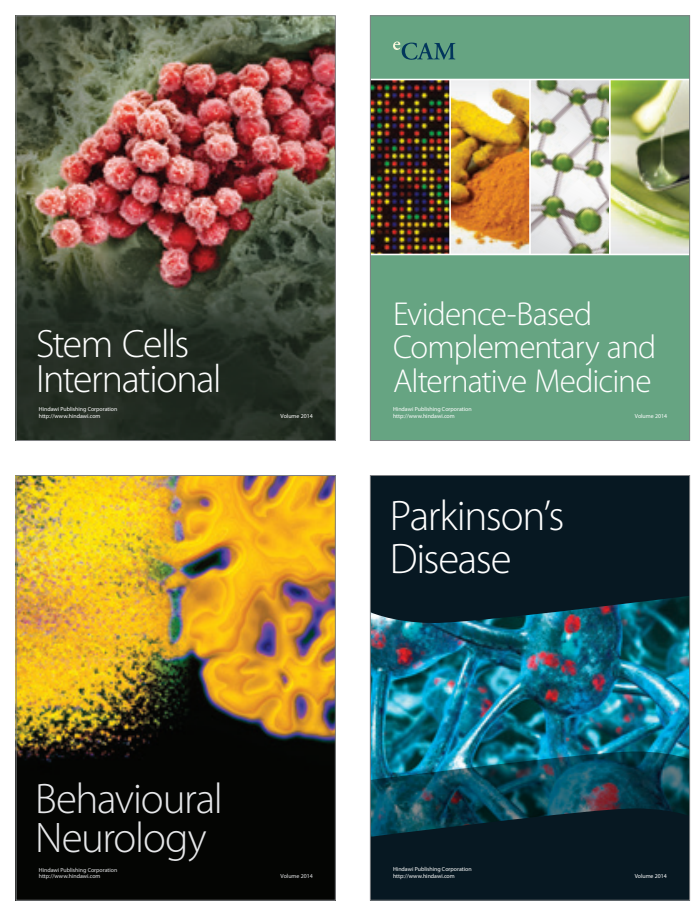

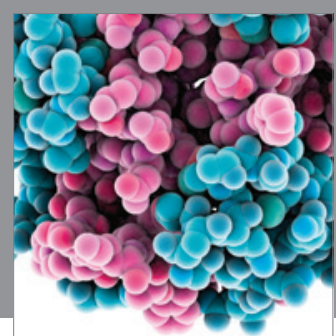

Journal of
Diabetes Research

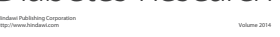

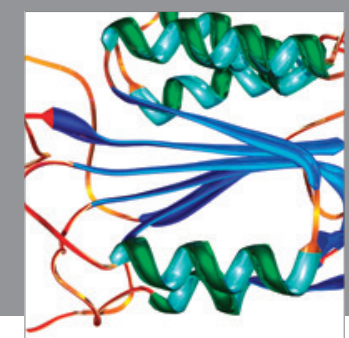

Disease Markers
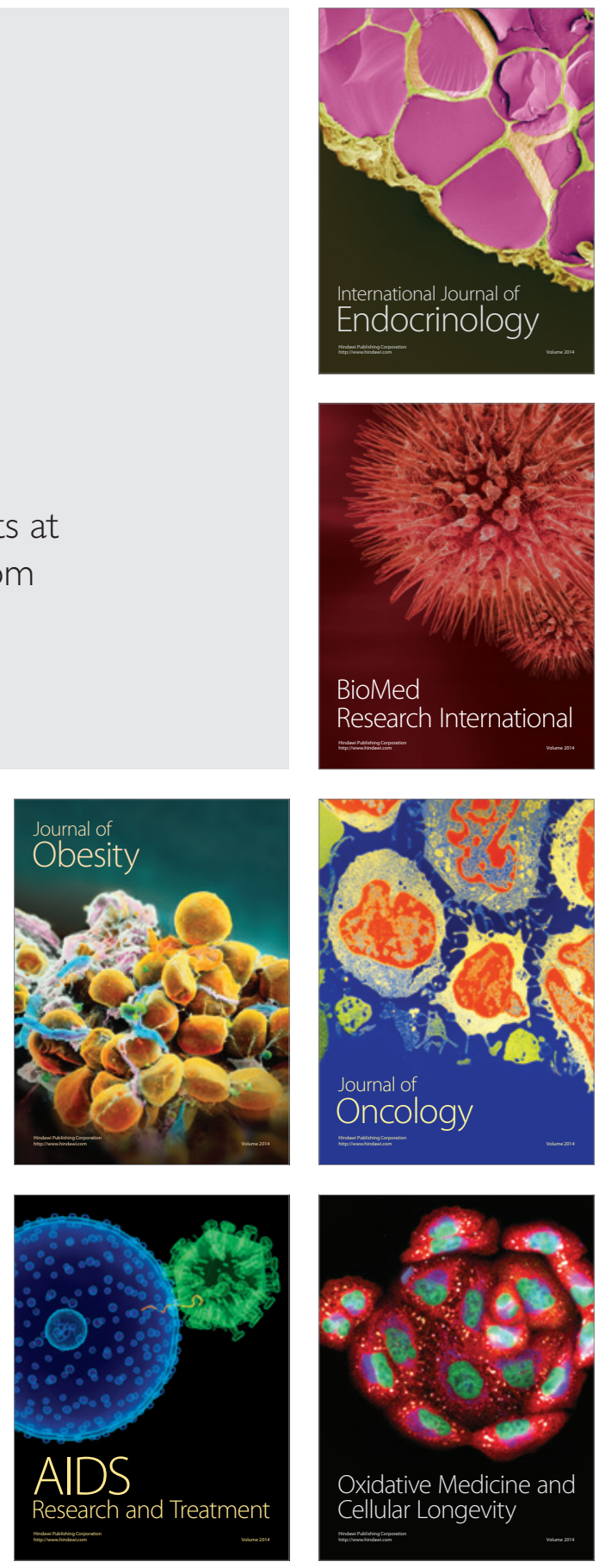